\title{
A Type-2 Fuzzy in Image Extraction for DICOM Image
}

\author{
D. Nagarajan ${ }^{1}$, M.Lathamaheswari ${ }^{2}$ \\ Department of Mathematics \\ Hindustan Institute of Technology \& Science \\ Chennai-603 103, India
}

\author{
J.Kavikumar ${ }^{3}$, Hamzha ${ }^{4}$ \\ Department of Mathematics and Statistics \\ Universiti Tun Hussein Onn \\ Malaysia
}

\begin{abstract}
Eradication of a desired portion of an image is a very important role in image processing and is also called feature extraction. This is mainly concern about reducing the number of possessions required to portray a large set of data and also reduce memory space requirement and power of data processing. Perfectly optimized feature extraction is an essential process for an effective design construction. Though there are many tools are available for extracting a feature, Type-2 Fuzzy Logic plays a vital role in producing good results. In this paper, weighted arithmetic operator is proposed using Yager triangular norms and proved the properties of the triangular norms using proposed operator. Also, the paper relates the properties to feature extraction. Also Brain has been extracted from patient MRI DICOM image using MATLAB based on Type-2 Fuzzy setting.
\end{abstract}

Keywords-Feature extraction; MRI image; type-2 fuzzy; MATLAB; triangular norms; mathematical properties

\section{INTRODUCTION}

Image processing is a mapping from image points to a new value by dealing a single point from original image and thereafter it will continue for group of points. Feature extraction is nothing but shape eradication which analyzes the contest of low level data to a known design of a desired shape Also it indicates verdict their location, direction and size. Minor particulars of the image such as dots and lines are called low level features whereas high level features are assembled on the elite of low level features to catch objects and bigger shapes in the image [1-4]. This shows the important steps involved in feature extraction.

In image processing number of points and their range makes a specific effect and the main components are grayscale, resolution, color, dynamic range and storage. Image processing and feature extraction is equitable to the illumination of the matching point in the location and generally its value is determined from the output. Image is a matrix of picture elements in square shape and is described by $M \times M n$-bit picture elements i.e., pixels. Here $M$ is the number of points through the axes and $n$ manages the number of values for brightness. Adopting $n$ bits provides a range of $2 n$ values, ordering from 0 to $2 n-1$ which is the brightness level and is generally presented as white and black. Smaller value of $n$ represents the decrement in image contrast [5, 910]. This part expresses the color contrast of the image.
Feature extraction is also meant for increasing pixel brightness to find the desired part of the image. In extracting a feature invariance property is to be strictly followed as this process should not vary according to the specified conditions it also implies the reliability and stability of the shape which is extracted. Hence it is necessary to choose the technology for feature extraction where there is a control over the parameters [11-12]. Hence choosing the technology for image extraction is very important to get better results.

The passion histogram exhibits how particular brightness levels are involved in an image and contrast of the image is calculated by the range of brightness levels. This technology plans the number of pixels with a specific level of brightness contrary to the level of brightness. All these contrary things can be dealt by fuzzy logic as it deals with uncertainty well. Generally images have uncertainty in the desired part and clear shape of it. Type-2 fuzzy logic plays an efficient role as it deals with upper and lower membership functions and foot print of uncertainty (the area between upper and lower membership functions) and its length represents uncertainty level of the image or part which is to be extracted [13]. This is the role of fuzzy logic under type-1 and type-2 fuzzy environments.

These technologies are the mathematical systems which provide an outstanding direction for better understanding about the process. Mathematica, Mathcad, MATLAB and Maple are some of the popular tools. Where in MATLAB, by giving instructions at the mandate line, the procedure will be operated and the outputs can be displayed as surfaces, graphs and images. Hence MATLAB under Type-2 fuzzy setting will provide a desired result. In this work, using Type-2 Fuzzy MATLAB, brain is extracted from MRI image. Even though the convenient measures the performance for the credential design analysis are not recognizable and difficult to derive, it helps to design a system which improves its performance measures for training data set and forecast the performance of the system for testing data set $[1,14]$.

Feature extraction of the image to an observable level is an essential key in advance of Content Based Image Retrieval systems. From texture, color and structure of the image, the low level optical features of an image can be separated and can be used while recovery to correlate concern image and other images in the database [12, 18]. This is the stage of acquiring information about image objects which is to be analyzed. On the basis of both quantitative and qualitative 
reasoning, the features may be determined where the ideas might be hypothetical from the expert which is modified into quantitative values. Color image is one of the universally used features due to its stability, efficiency and computational simplicity $[12,15-16]$. Hence color images have potential of clarity of the features which are to be extracted.

A color image based on mathematics is called a digital image and it consists of color information for every picture element. It is also a binary image which has only two possible values for every pixel and is reserved as a single bit 0 or 1 . To design a binary image, a threshold intensity value needs to be selected. Pixels with greater intensity than the threshold value are switch to 0 (black) whereas when the intensity value less than the threshold level are switch to 1 (White) and hence the image is converted as a binary image. Gray scale images are having a range of darkness without possible color and used as a fewer information needs to be contributed for each and every pixel. The calculation of mathematical captions could contribute more information about the parameters of morphology but they are not interpreted easily [17-20, 30]. Therefore the procedure for morphology is supposed to be taken care for getting a good feature.

Medical images are generally uncertain in nature. Though there are many methodologies are available to extract the feature from the image, Fuzzy logic (Type-1) helps to extract the feature in an efficient manner but the membership functions are crisp which lies between 0 and 1. MATLAB on Type-2 fuzzy setting provides an optimized result as it handles more uncertainties based on the Footprint of uncertainty, the area between upper and lower membership functions.

From the previous proposed solutions image extraction has not been done using interval type-2 fuzzy logic for feature extraction of the brain from patient MRI. This is the shortcoming of the previous studies and the motivation of the present study. Throughout the paper type-2 fuzzy has been considered as interval type-2 fuzzy environment.

In this paper, the mathematical properties of triangular norms has been proved as it represents the essential qualities of image processing and brain is extracted from patient MRI which is taken from our experimental data using MATLAB and provided the 3D image of an extracted brain with the key components such as DICOM image of a patient MRI, interval type-2 mat lab coding for feature extraction.

\section{REVIEW OF LITERATURE}

The authors of, [1] proposed the idea of linguistic variable and its application in the field of approximate reasoning. The researcher in [2] proposed different classes of fuzzy operators. The researcher in [3] proposed novel aggregations operators under probabilistic fuzzy environment. The researcher in [4] reviewed aggregation connectives under fuzzy environment. The researcher in [5] introduced theory of t-norms and inference methods under fuzzy setting. The researcher in [6] designed fuzzy systems and derived aggregation operators. The researcher in [7] discussed about imprecise reasoning for interval based data with the support of fuzzy and rough sets. The researcher in [8] proposed aggregation operators in detail and applied them video querying.
The researcher in [9] introduced fuzzy image processing using Dubois and Prade aggregation operators. The researcher in [10] proposed a computer based system on hand written records to hold forensic studies. The researcher in [11] proposed means with weight using triangular co norms. The researcher in [12] introduced elementary minimum and maximum operational laws for fuzzy numbers. The researcher in [13], the author aggregated the information collected using aggregation operations. The researcher in [14] applied fuzzy relational equations for Lossy image compression and reconstruction. The researcher in [15] proposed OWA operators with imprecise weights based on type-1 fuzzy. The researcher in [16] analyzed aggregation functions. The researcher in [17] introduced exact computations of protracted logical operations based on uncertain truth values. The researcher in [18] examined and compared different approaches over edge detection.

The researcher in [19] applied morphological operators in image analysis on uninorms. The researcher in [20] proposed novel aggregation operators for the method of active learning. The researcher in [21] explained and derived aggregation operators in detail. The researcher in [22] proposed triangular interval type-2 aggregation operators using Frank triangular norms and applied them in a decision making method. The researcher in [23] proposed fuzzy metric spaces. The researcher in [24], the author applied idea of fuzzy methodology in medical image processing. The researcher in [25] examined collection of information and the related aggregation operators. The researcher in [26] reviewed recent year applications of image processing under type-2 fuzzy.

In [27] the imitation of edge detection of an image using MATLAB with fuzzy logic is done. The researcher in [28] reviewed the role of type-2 fuzzy in the field of Bio medicine. The researcher in [29] proposed edge detection method for a digital image using fuzzy logic. The researcher in [30] proposed a methodology for edge detection for a DICOM image using aggregation operators under type-2 fuzzy. The researcher in [31] implemented a methodology for image fusion using intuitionistic fuzzy logic. The researcher in [32] reviewed about fuzzy controllers to sustain the stability of the system using type-2 fuzzy. The researcher in [33] analyzed surface of the material on curve features of digital images using fuzzy logic. The researcher in [34] proposed 3D version of brain visualization using machine learning. The researcher in [35] proposed block processing and edge detection on DICOM image. The researcher in [36] introduced denoising of the image using LU decomposition method and feature extraction using GLCM.

From this review it is found that there is no contribution of research for image extraction from a DICOM image using interval Type- 2 fuzzy logic. This is the motivation of the present work.

\section{BASIC DEFINITIONS}

The following basic concepts are given for the better understanding of the paper.

\section{A. Aggregation Operator [22]}


Let $\left(M_{\alpha}\right)_{\alpha \in[0,1]}$ be a group of aggregation operators (AOs) which is non-decreasing. If $\mathrm{A}$ is an $\mathrm{AO}$ then

$M_{A}: \bigcup_{n \in N}[0,1]^{n} \rightarrow[0,1]$.

Triangular Interval Type-2 Fuzzy Set (TIT2FS) [22]

The membership function (MFs) are developed using triangular fuzzy number in IT2FS called TIT2FS. In IT2FS, upper and lower MFs represented by a triangular fuzzy number $\bar{M}=\left\langle\left[\underline{l_{M}}, \overline{l_{M}}\right], c_{M},\left[\underline{r_{M}}, \overline{r_{M}}\right]\right\rangle$ called TIT2FS and are defined by

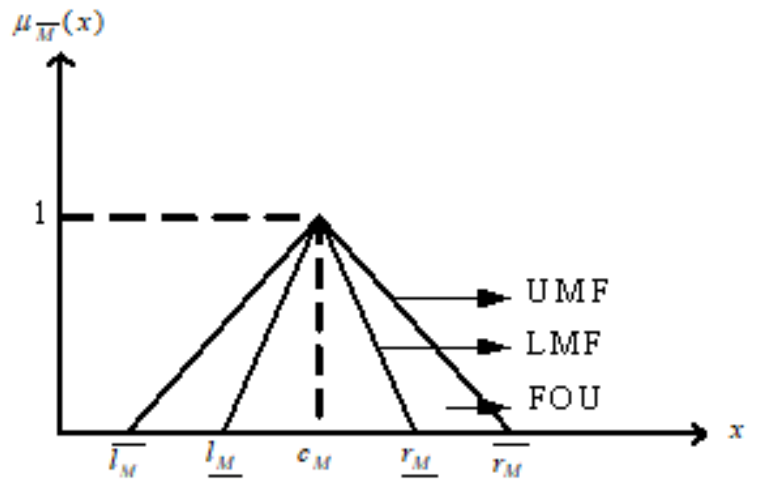

Fig. 1. TIT2FS.



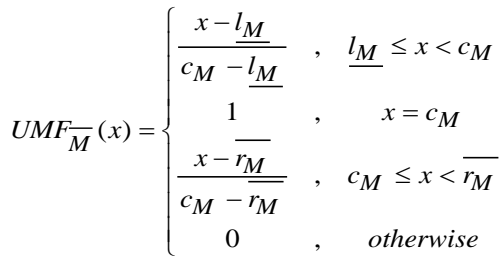

where $\underline{l_{M}}, \overline{l_{M}}, c_{M}, \underline{r_{M}}, \overline{r_{M}}$ are the measuring points on TIT2FS satisfying $0 \leq l_{M} \leq \overline{l_{M}} \leq c_{M} \leq \underline{r_{M}} \leq \overline{r_{M}} \leq 1$. If we consider $x$ as a set of real numbers, a TIT2FS in $x$ is called TIT2FN. The FOU is the area between lower and upper membership functions in figure 1. If $l_{\underline{M}}=\bar{l}_{M}, r_{M}=\overline{r_{M}}$, then $U M F_{\bar{M}}(x)=$ $L M F_{\bar{M}}(x)$ for all the values of $x$ in $X$, then the TIT2FS will become Type-1 case. Here FOU is the footprint of Uncertainty.

B. Ranking Formula for TIT2FN [22]
Let $\bar{M}=\langle[A, B], C,[D, E]\rangle$

where $A=\underline{l_{M}}, B=\overline{l_{M}}, C=c_{M}, D=\underline{r_{M}}, E=\overline{r_{M}}$ be the TIT2FN. The ranking value is defined by

$\operatorname{Rank}(\bar{M})=\left(\frac{A+E}{2}+1\right) \times \frac{A+B+D+E+4 C}{8}$

C. Yager Triangular Norms [5]

$\stackrel{\otimes}{Y}$ is Yager product (T Norm) and $\stackrel{\oplus}{Y}$ is a Yager sum

( $\mathrm{T}$ conorm) and are defined as follows.

$\underset{Y}{r \otimes s}=\max \left(1-\left[(1-r)^{\eta}+(1-s)^{\eta}\right]^{\frac{1}{\eta}}, 0\right), \eta>0$, for all $r, s \in[0,1]^{2}$

(4)

$r \underset{Y}{\oplus} s=\min \left(\left(r^{\eta}+s^{\eta}\right)^{\frac{1}{\eta}}, 1\right), \eta>0$, for all $r, s \in[0,1]^{2}$

D. Triangular Interval Type-2 Fuzzy Yager Weighted

Arithmetic (TIT2FYWA) Operator [22]

Consider a set of TIT2FNs and the operator TIT $2 F Y W A_{\varepsilon}: \Omega^{n} \rightarrow \Omega$ is defined by TIT2FYWA $A_{\varepsilon}\left\langle\overline{M_{1}}, \overline{M_{2}}, \ldots, \overline{M_{n}}\right\rangle=\varepsilon_{1} \bullet \overline{M_{1}} \underset{Y}{\oplus} \varepsilon_{2} \bullet \overline{M_{2}} \underset{Y}{\oplus} \ldots \underset{Y}{\oplus} \varepsilon_{n} \bullet \overline{M_{n}}$ and its weight vector is $\varepsilon=\left(\varepsilon_{1}, \varepsilon_{2}, \ldots, \varepsilon_{n}\right)^{T}$ and the sum of the weight vectors is equal to 1 , when $\varepsilon=(1 / n, 1 / n, \ldots, 1 / n)^{T}$, triangular interval type-2 fuzzy Yager weighted arithmetic operator will become triangular interval type-2 fuzzy Yager arithmetic averaging operator of dimension $n$ and is defined by.

$\operatorname{TIT}_{2 F Y A}\left(\overline{M_{1}}, \overline{M_{2}}, \ldots, \overline{M_{n}}\right)=\frac{1}{n} \bullet\left(\overline{M_{1}} \underset{Y}{\oplus} \overline{M_{2}}, \ldots, \underset{Y}{\oplus} \overline{M_{n}}\right)$

E. Triangular Interval Type-2 Fuzzy Yager Weighted Geometric (TIT2FYWG) Operator [22]

Let $\left.\bar{M}=\left(\left[\underline{l_{M_{p}}}, \overline{l_{M_{p}}}\right], c_{M_{p}}, \underline{{ }^{\prime} M_{p}}, \overline{r_{M_{p}}}\right]\right), \quad p=1,2, \ldots, n$ be a set of TIT2FNs. Triangular Interval Type-2 fuzzy Yager Weighted Geometric Mean Operator (TIT2FYWA), TIT2FYWG: $\varepsilon^{\mathrm{n}} \rightarrow \varepsilon$ is $\quad \operatorname{TIT2FYWG}_{\varepsilon}\left(\overline{M_{1}}, \overline{M_{2}}, \ldots, \overline{M_{n}}\right)=\overline{M_{1}{ }^{Y} \varepsilon_{1}}{\underset{Y}{M_{2}} \bar{M}^{Y} \varepsilon_{2}}_{Y}^{\otimes} \ldots{ }_{Y}^{\otimes} \overline{M_{n}^{Y}} \varepsilon^{\varepsilon_{n}} \quad$.Here also sum of all weight vectors is equal to 1 , when $\varepsilon=(1 / n, 1 / n, \ldots, 1 / n)^{T}$, triangular interval type-2 fuzzy Yager weighted arithmetic operator will become triangular interval type-2 fuzzy Yager geometric averaging operator of 
dimension $\mathrm{n}$ and is defined by $\operatorname{TIT}_{2} F Y G A_{\varepsilon}\left(\overline{M_{1}}, \overline{M_{2}}, \ldots, \overline{M_{2}}\right)=$ $\frac{1}{n}\left(\overline{M_{1}} \underset{Y}{\otimes} \overline{M_{2}} \underset{Y}{\otimes}, \ldots, \underset{Y}{\otimes} \overline{M_{n}}\right) \dot{Y} 1 / n$

\section{F. Feature Extraction [18]}

The task of feature extraction converts affluent content of images into different content features and a process of producing features to be used in the classification and selection effort. It is the second accent of classification of the image and helps to reduce the number of features supplied to the categorization task. After the selection of desired feature, that will be used in the task and failing are discarded. Even though a reduction is desirable in dimensionality, increment in inaccuracy has to be along with selective power of classifiers. This technique is useful when the size of the image is large and extracted feature is helpful for quick completion of the task like matching of the images and recovery.

\section{G. Class of Features [19]}

An operation of more than one dimension which designates few perceptible properties of the image/ body is called a feature and it measures some important components of the object. The following are the different features.

1) General features: Texture, color and shape are the purpose free features and called general features. According to the conceptual level, further they are divided into the following.

- Pixel-level features, which are estimated at every pixel such as location and color.

- Local Features, features estimated over the decision of class of the image line on image bisection and detection of edge.

- Global Features, features steered over the complete image impartially.

2) Domain explicit features: These features are application dependent such as finger prints, human faces and visionary features which are generally a fusion of low level features for a particular domain.

\section{H. Color Feature [19]}

Color feature is the visual feature and is extensively used in image recovery. Stability, efficiency, simplicity in implementation and computationally as well and low storage capacity are the advantages of color features.

\section{Morphological Operators [19]}

Morphological operators convert the original image into another one by interacting with the other images of the structural elements like absolute size and shape and it provides a systematic approach for characterization in many applications like object segmentation, edge detection and suppression of noise. The goal of Fuzzy mathematical morphology is to develop the binary morphological operators to gray level images.

\section{J. Mathematical Morphology (MM) [19]}

MM is a group of operations. Erosion, dilation, opening and closing are the essential morphological operations on the image pixels. To segregate bright and dark structures than the neighboring features, opening and closing transforms can be used. General methodology has been introduced for fuzzy dilation, erosion, opening and closing.

Dilation is the mechanism of increasing the maximum value in the window thereafter the brightness of the image will be increased and the image objects will be extended as well by modifying the value from 0 to 1 . Erosion is the reverse process of dilation, here the image becomes darker than the original and deals the image by converting the pixel value from 1 to 0 .

For getting stabilized gray scale images, morphological openings and closings are playing a vital role in image processing. These mathematical morphological operations are similar to the design of set theory and its operations as well. At this junction, fuzzy set theory plays an efficient role in mathematical morphology as the images are uncertain in nature.

\section{K. Morphological Caption [19]}

Area, volume, perimeter, gray levels, density, maximum, minimum average, standard deviation of major and minor axes, location, unusualness, point of restriction and centroid are the morphological descriptors or captions in image processing.

\section{Projections [19]}

Generally in image processing, there are two types projections are available on binary images such as horizontal and vertical projections. This process scan from left side of each line and records the pixel changes from 0 to 1 and again to 0 where the number of changes is independent of the pixels. Stability can be expected even in noisy condition in this process. After obtaining associated components, progress of the pixel values from 0 to 1 or vice versa, need to be checked horizontally whereas background area have less progress or transitions.

If the allotted amount of changes of each row lies between two thresholds such as low and high then that row will be considered as a desired area. Next vertical transitions will be considered to find the exact location of the feature which is to be extracted by inquiring the length and height of the feature and their ratio and adequate number of pixels in that area [13].

\section{PROposed Operational LAWS}

Let $\bar{F}, \bar{F}_{1}, \bar{F}_{2}$ be three TIT2FNs and $\chi>0$, then we define their operational laws as follows.

\section{A. Addition Consider}

$$
U_{1}=\underset{t=1}{\operatorname{aot}}\left(\underline{l_{F_{t}}}\right), V_{1}=\underset{t=1}{\operatorname{aot}}\left(\overline{l_{F_{t}}}\right), W_{1}=\underset{t=1}{\operatorname{aot}}\left(c_{M_{t}}\right), X_{1}=\underset{t=1}{\operatorname{aot}}(\underbrace{r_{t}}_{r_{t}}), Y_{1}=\underset{t=1}{\operatorname{aot}}\left(\overline{r_{F_{t}}}\right) .
$$

where aot $=$ sum of the terms 
$\overline{F_{1}} \oplus \overline{F_{2}}=\left(\left[\min \left(a_{1}^{\frac{1}{\chi}}, 1\right), \min \left(b_{1}^{\frac{1}{\chi}}, 1\right)\right], \min \left(c_{1}^{\frac{1}{\chi}}, 1\right)\right.$,

$\left[\min \left(d_{1}^{\frac{1}{\chi}}, 1\right), \min \left(e^{\frac{1}{\chi}}, 1\right)\right]$

\section{B. Multiplication Consider}

$U_{2}=\underset{t=1}{a \operatorname{aot}}\left(\underline{l_{F_{t}}}\right)^{\chi}, V_{2}=\underset{t=1}{\operatorname{aot}}\left(\overline{l_{F_{t}}}\right)^{\chi}, W_{2}=\underset{t=1}{\operatorname{aot}}\left(c_{F_{t}}\right)^{\chi}$,

$X_{2}=\underset{t=1}{\operatorname{aot}}\left(\underline{r_{F_{t}}}\right)^{\chi}, Y_{2}=\underset{t=1}{\operatorname{aot}}\left(\overline{r_{F_{t}}}\right)^{\chi}$.

$\overline{F_{1}} \oplus \overline{F_{2}}=\left\{\left[\min \left(U_{2}{ }^{\frac{1}{\chi}}, 1\right), \min \left(V_{2}^{\frac{1}{\chi}}, 1\right)\right], \min \left({W_{2}}^{\frac{1}{\chi}}, 1\right)\right.$,

$\left.\left[\min \left(X_{2}^{\frac{1}{\chi}}, 1\right), \min \left(Y_{2}^{\frac{1}{\chi}}, 1\right)\right]\right\}$

C. Multiplication by an Ordinary Number Consider

$U=\underline{l_{F}}, V=\overline{l_{F}}, W=c_{F}, X=\underline{r_{F}}, Y=\overline{r_{F}}$

$g \bullet \bar{F}=\left\{\left[\min \left\langle U^{\frac{g}{\chi}}, 1\right\rangle, \min \left\langle V^{\frac{g}{\chi}}, 1\right\rangle, \min \left\langle W^{\frac{g}{\chi}}, 1\right\rangle\right.\right.$,

$\left[\min \left\langle X^{\frac{g}{\chi}}, 1\right\rangle, \min \left\langle Y^{\frac{g}{\chi}}, 1\right\rangle\right\}$.

\section{Power Consider}

$U_{3}=l_{F}, V_{3}=\overline{l_{F}}, W_{3}=c_{F}, X_{3}=r_{F}, Y_{3}=\overline{r_{F}}$

$\bar{F}^{g}=\left\{\left[\max \left(1-\left[a_{3} \chi\right]^{g / \chi}, 0\right), \max \left(1-\left[b_{3}^{\chi}\right]^{g / \chi}, 0\right)\right]\right.$,

$\max \left(1-\left[c_{3}\right]^{g / \chi}, 0\right)$

$\left.\left[\max \left(1-\left[d_{3} \chi\right]^{g / \chi}, 0\right), \max \left(1-\left[e_{3}\right]^{g / \chi}, 0\right)\right]\right\}$

\section{PROPOSED THEOREMS}

Here the mathematical properties of aggregation properties are proved for TIT2FN using TIT2WA operator which represents the essential qualities of the image processing such as idempotent ability, stability and image contrast.
Let TIT2FNs $\bar{F}=\left(\left[l_{F_{t}}, \overline{l_{F_{t}}}\right], c_{F_{t}},\left[r_{F_{t}}, \overline{r_{F_{t}}}\right]\right), t=1,2, \ldots, n$, where $0 \leq \underline{l_{F}} \leq \overline{l_{F}} \leq c_{F} \leq \underline{r_{F}} \leq \overline{r_{F}} \leq 1$ be a collection of TIT2FNs.

\section{A. Theorem}

The aggregation value of these fuzzy numbers using TIT2FYWG operator is again a TIT2FN and

$$
\begin{aligned}
& \operatorname{TIT}_{2 W A_{\rho}}\left\langle\overline{F_{1}}, \overline{F_{2}}, \ldots, \overline{F_{n}}\right\rangle=\left\{\left[\min \left(\left[U_{n} \chi\right]^{\rho_{t} / x}, 1\right), \min \left(\left[V_{n}\right]^{\rho_{t} / x}, 1\right)\right],\right. \\
& \left.\min \left(\left[W_{n}\right]^{\rho_{t} / x}, 1\right),\left[\min \left(\left[X_{n}^{\eta}\right]^{\rho_{t} / x}, 1\right), \min \left(\left[Y_{n}\right]^{\rho_{t} / x}, 1\right)\right]\right\}
\end{aligned}
$$

Where the weight vector is $\rho=\left(\rho_{1}, \rho_{2}, \ldots, \rho_{n}\right)^{T}, \rho_{n} \geq 0$, the sum of the weight vectors is equal to 1 .

Proof:

The aggregation value of these fuzzy numbers using TIT2FYWG operator is again a TIT2FN and

$$
\begin{aligned}
& \operatorname{TIT}_{2} \mathrm{WA}_{\rho}\left\langle\overline{F_{1}}, \overline{F_{2}}, \ldots, \overline{F_{n}}\right\rangle=\left\{\left[\min \left(\left[U_{n} \chi\right]^{\rho_{t} / \chi}, 1\right), \min \left(\left[V_{n}{ }^{\eta}\right]^{\rho_{t} / x}, 1\right)\right],\right. \\
& \left.\min \left(\left[W_{n}{ }^{\eta}\right]^{\rho_{t} / x}, 1\right),\left[\min \left(\left[X_{n}\right]^{\rho_{t} / \chi}, 1\right), \min \left(\left[Y_{n}{ }^{\eta}\right]^{\rho_{t} / x}, 1\right)\right]\right\},
\end{aligned}
$$

Where the weight vector is $\rho=\left(\rho_{1}, \rho_{2}, \ldots, \rho_{n}\right)^{T}, \rho_{n} \geq 0$, the sum of the weight vectors is equal to 1 .

Here use mathematical induction method.

Case (i): For $n=2$

Consider,

$U_{1}=\left(\underline{l_{F_{1}}}\right)^{\chi}, V_{1}=\left(\overline{l_{F_{1}}}\right)^{\chi}, W_{1}=\left(c_{F_{1}}\right)^{\chi} \quad X_{1}=\left(\underline{r_{F_{1}}}\right)^{\chi}, Y_{1}=\left(\overline{r_{F_{1}}}\right)^{\chi}$

Using multiplication operation

$$
\begin{aligned}
& g \bullet \overline{F_{1}}=\left\{\left[\min \left\langle U_{1}^{\frac{g}{\chi}}, 1\right\rangle, \min \left\langle V_{1}^{\frac{g}{\chi}}, 1\right\rangle\right], \min \left\langle W_{1}^{\frac{g}{\chi}}, 1\right\rangle, .\right. \\
& \left.\left[\min \left\langle X_{1}^{\frac{g}{\chi}}, 1\right\rangle, \min \left\langle Y_{1}^{\frac{g}{\chi}}, 1\right\rangle\right]\right\}
\end{aligned}
$$

Consider,

$U_{2}=\left(\underline{l_{F_{2}}}\right)^{\chi}, V_{2}=\left(\overline{l_{F_{2}}}\right)^{\chi}, W_{2}=\left(c_{F_{2}}\right)^{\chi}, X_{2}=\left(\underline{r_{F_{2}}}\right)^{\chi}, Y_{2}=\left(\overline{r_{F_{2}}}\right)^{\chi}$ 


$$
\begin{aligned}
& g \bullet \overline{F_{2}}=\left\{\left[\min \left\langle U_{2}^{\frac{g}{\chi}}, 1\right\rangle, \min \left\langle V_{2}^{\frac{g}{\chi}}, 1\right\rangle, \min \left\langle W_{2}^{\frac{g}{\chi}}, 1\right\rangle,\right.\right. \\
& {\left[\min \left\langle X_{2}^{\frac{g}{\chi}}, 1\right\rangle, \min \left\langle Y_{2}^{\frac{g}{\chi}}, 1\right\rangle\right] .} \\
& r \underset{Y}{\oplus} s=\min \left(\left(r^{\eta}+s^{\eta}\right)^{\frac{1}{\eta}}, 1\right), \eta>0, \text { for all } r, s \in[0,1]^{2} \\
& \operatorname{TIT} 2 F W A_{\rho}\left(\overline{F_{1}}, \overline{F_{2}}\right)=\rho_{1} \bullet \overline{F_{1}} \oplus \rho_{1} \bullet \overline{F_{2}} \\
& =\left\{\left[\min \left(\left[\begin{array}{c}
2 \\
\operatorname{aot} t \\
t=1
\end{array}\left(\min \left(\left(U_{2}\right)^{\frac{\rho_{t}}{\chi}}\right), 1\right)\right], 1\right), \min \left(\left[\begin{array}{c}
2 \\
\operatorname{aot} \\
t=1
\end{array}\left(\min \left(\left(V_{2}\right)^{\frac{\rho_{t}}{\chi}}\right), 1\right)\right], 1\right],\right.\right. \\
& \min \left(\left[\begin{array}{c}
2 \\
\operatorname{aot} \\
p=1
\end{array}\left(\min \left(\left(W_{2}\right)^{\frac{\rho_{t}}{\chi}}\right), 1\right)\right], 1\right) \\
& \left.\left[\min \left(\left[\begin{array}{c}
2 \\
\operatorname{aot} \\
t=1
\end{array}\left(\min \left(\left(X_{2}\right)^{\frac{\rho_{t}}{\chi}}\right), 1\right)\right], 1\right), \min \left(\left[\begin{array}{c}
2 \\
\operatorname{aot} \\
1=1
\end{array}\left(\min \left(\left(Y_{2}\right)^{\frac{\rho_{t}}{\chi}}\right), 1\right)\right], 1\right)\right]\right\} \text {. }
\end{aligned}
$$

Consider,

$$
\begin{aligned}
& U_{4}=\left(1-\underline{l_{M_{p}}}\right)^{\chi}, V_{4}=\left(1-\overline{l_{M_{t}}}\right)^{\chi}, W_{4}=\left(1-c_{M_{t}}\right)^{\chi} \text {, } \\
& X_{4}=\left(1-\underline{r_{M_{t}}}\right)^{\chi}, Y_{4}=\left(1-\overline{r_{M_{t}}}\right)^{\chi} .
\end{aligned}
$$

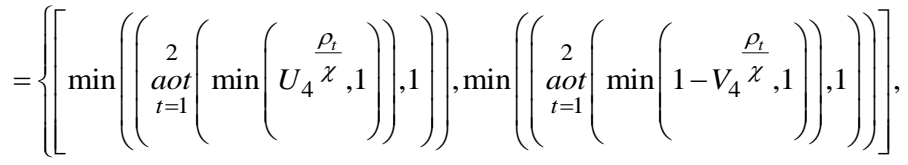

$$
\begin{aligned}
& \min \left(\left(\underset{t=1}{\operatorname{aot}}\left(\min \left(W_{4}^{\frac{\rho_{t}}{\chi}}, 1\right)\right), 1\right)\right)
\end{aligned}
$$

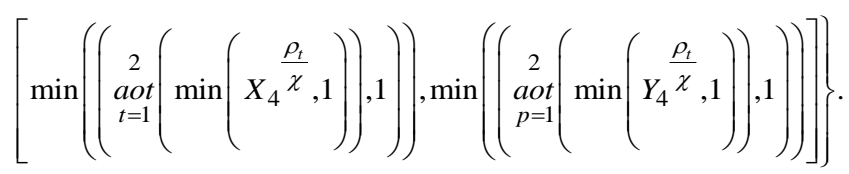

$$
\begin{aligned}
& =\left\{\left[\min \left[U_{2}{ }^{\frac{\rho_{t}}{\chi}}, 1\right], \min \left[V_{2}^{\frac{\rho_{t}}{\chi}}, 1\right], \min \left[{W_{2}}^{\frac{\rho_{t}}{\chi}}, 1\right],\right.\right. \\
& \left.\left[\min \left[X_{2}^{\frac{\rho_{t}}{\chi}}, 1\right], \min \left[Y_{2}^{\frac{\rho_{t}}{\chi}}, 1\right]\right]\right\}
\end{aligned}
$$

For $n=k$,

$$
\begin{aligned}
& U_{k}=\underset{t=1}{a \operatorname{aot}}\left(1-\underline{l_{F_{t}}}\right)^{\chi}, V_{k}=\underset{t=1}{\operatorname{aot}}\left(1-\overline{l_{F_{t}}}\right)^{\chi}, W_{k}=\underset{t=1}{\operatorname{aot}}\left(1-c_{F_{t}}\right)^{\chi}, \\
& X_{k}=\underset{t=1}{\operatorname{aot}}\left(1-\underline{r_{F_{t}}}\right)^{\chi}, Y_{k}=\underset{t=1}{\operatorname{aot}}\left(1-\overline{r_{F_{t}}}\right)^{\chi} \\
& \operatorname{TIT} 2 W A_{\rho}\left\langle\overline{F_{1}}, \overline{F_{2}}, \ldots, \overline{F_{k}}\right\rangle
\end{aligned}
$$

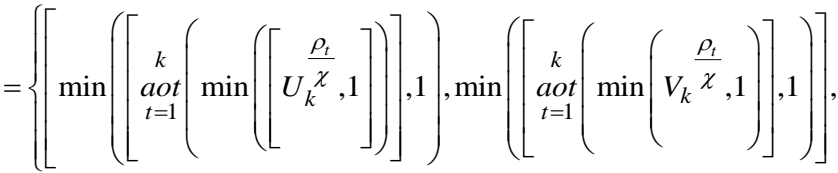

$$
\begin{aligned}
& \min \left(\left[\begin{array}{c}
k \\
\operatorname{aot} \\
t=1
\end{array}\left(\min \left(W_{k}^{\frac{\rho_{t}}{\chi}}, 1\right)\right], 1\right)\right.
\end{aligned}
$$

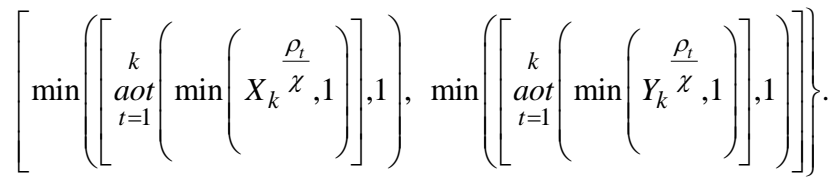

$$
\begin{aligned}
& =\left\{\left[\min \left[U_{k}^{\frac{\rho_{t}}{\chi}}, 1\right], \min \left[V_{k}^{\frac{\rho_{t}}{\chi}}, 1\right], \min \left[W_{k}^{\frac{\rho_{t}}{\chi}}, 1\right]\right.\right. \text {, }
\end{aligned}
$$$$
\left.\left[\max \left[X_{k}^{\frac{\rho_{t}}{\chi}}, 1\right], \max \left[Y_{k}^{\frac{\rho_{t}}{\chi}}, 1\right]\right]\right\}
$$$$
\text { For } n=k+1 \text {, }
$$$$
\operatorname{TIT} 2 W A_{\rho}\left\langle\overline{F_{1}}, \overline{F_{2}}, \ldots, \overline{F_{k}}\right\rangle \oplus\left\langle\overline{F_{k+1}}\right\rangle
$$

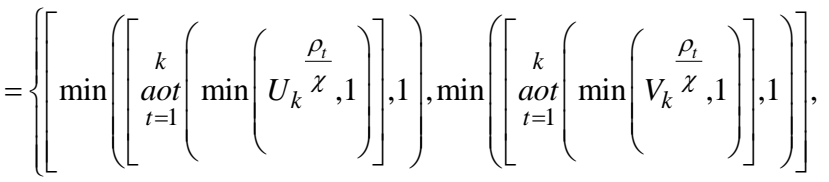$$
\min \left(\left[\underset{t=1}{\operatorname{aot}}\left(\min \left(W_{k}^{\frac{\rho_{t}}{\chi}}, 1\right)\right], 1\right)\right.
$$$$
\left[\min \left(\left[\begin{array}{c}
k \\
\operatorname{aot} \\
t=1
\end{array}\left(\min \left(X_{k}^{\frac{\rho_{t}}{\chi}}, 1\right)\right], 1\right), \min \left(\left[\underset{p=1}{k}\left(\min \left(Y_{k}^{\frac{\rho_{t}}{\chi}}, 1\right)\right], 1\right)\right]\right\} .\right.
$$$$
\oplus\left\{\min \left(U_{k+1}^{\frac{\rho_{k+1}}{\chi}}, 1\right), \min \left(V_{k+1}^{\frac{\rho_{k+1}}{\chi}}, 1\right)\right], \min \left(W_{k+1}^{\frac{\rho_{k+1}}{\chi}}, 1\right),
$$ 


$$
\begin{aligned}
& \left.\left[\max \left(X_{k+1}^{\frac{\rho_{k+1}}{\chi}}, 1\right), \max \left(Y_{k+1}^{\frac{\rho_{k+1}}{\chi}}, 1\right)\right]\right\}
\end{aligned}
$$

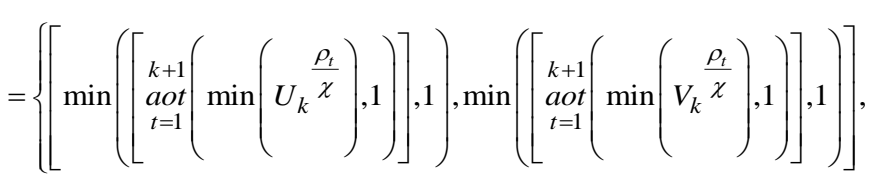

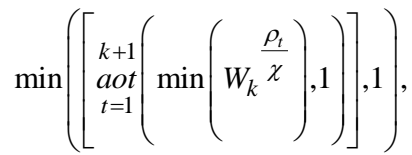

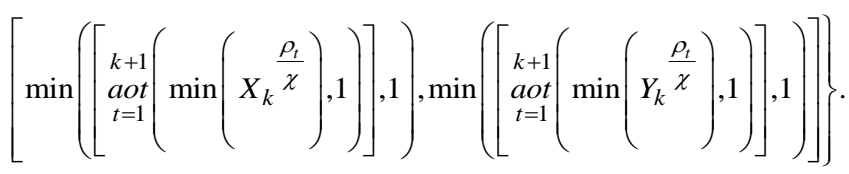

$$
\begin{aligned}
& =\left\{\left[\min \left[U_{k+1}^{\frac{\rho_{t}}{\chi}}, 1\right], \min \left[V_{k+1}^{\frac{\rho_{t}}{\chi}}, 1\right], \min \left[W_{k+1}^{\frac{\rho_{t}}{\chi}}, 1\right],\right.\right. \\
& \left.\left[\min \left[X_{k+1}^{\frac{\rho_{t}}{\chi}}, 1\right], \min \left[Y_{k+1}^{\frac{\rho_{t}}{\chi}}, 1\right]\right]\right\}
\end{aligned}
$$

Hence the result holds for all the values of $n$.

\section{B. Theorem (Idempotency)}

If $\overline{F_{t}}=\bar{F}$ for all the values of $\mathrm{t}$ then

$$
\operatorname{TIT} 2 W A_{\rho}\left\langle\overline{F_{1}}, \overline{F_{2}}, \ldots, \overline{F_{n}}\right\rangle=\bar{F} \text {. }
$$

Proof:

By theorem A,

$\operatorname{TIT} 2 W A_{\rho}\left\langle\overline{F_{1}}, \overline{F_{2}}, \ldots, \overline{F_{n}}\right\rangle=\left\{\left[\min \left(U_{n}^{\frac{\rho_{t}}{\chi}}, 1\right), \min \left(V_{n}^{\frac{\rho_{t}}{\chi}}, 1\right)\right], \min \left(W_{n}^{\frac{\rho_{t}}{\chi}}, 1\right)\right.$,

$\left.\left[\min \left(X_{n}^{\frac{\rho_{t}}{\chi}}, 1\right), \min \left(Y_{n}^{\frac{\rho_{t}}{\chi}}, 1\right)\right]\right\}$

$\operatorname{TIT} 2 W A_{\rho}\left\langle\overline{F_{1}}, \overline{F_{2}}, \ldots, \overline{F_{n}}\right\rangle$

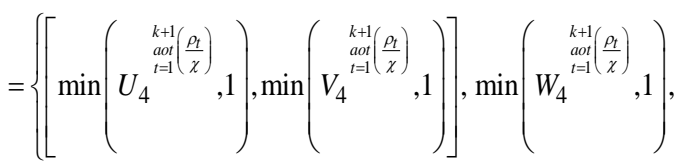

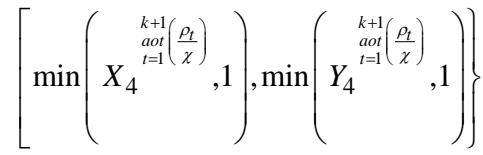

$=\left\{\left[\min \left(U_{5}^{\frac{1}{\chi}}, 1\right), \min \left(V_{5}^{\frac{1}{\chi}}, 1\right)\right], \min \left(W_{5}^{\frac{1}{\chi}}, 1\right)\right.$,

$\left.\left[\min \left(X_{5}^{\frac{1}{\chi}}, 1\right), \min \left(Y_{5}^{\frac{1}{\chi}}, 1\right)\right]\right\}$

$=\left\{\left[U_{3}, V_{3}, W_{3}, X_{3}, Y_{3}\right]\right\}=\{[U, V], W,[X, Y]\}=\bar{F}$

C. Theorem

If $f>0$ for all the values of $\mathrm{p}$ then

$\operatorname{TIT} W A_{\rho}\left(f \bullet \overline{F_{1}}, f \bullet \overline{F_{2}}, \ldots, f \bullet \overline{F_{n}}\right)=f \bullet \operatorname{TIT} 2 W A_{\rho}\left(\overline{F_{1}}, \overline{F_{2}}, \ldots, \overline{F_{n}}\right)$

Proof: Using,

$f \bullet \bar{F}=\left\{\left[\min \left(U_{5}^{\frac{f}{\chi}}, 1\right), \min \left(V_{5}^{\frac{f}{\chi}}, 1\right)\right], \min \left(W_{5}^{\frac{f}{\chi}}, 1\right)\right.$,

$\left.\left[\min \left(X_{5}^{\frac{f}{\chi}}, 1\right), \min \left(Y_{5}^{\frac{f}{\chi}}, 1\right)\right]\right\}$

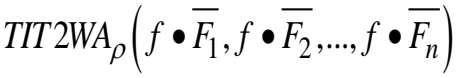

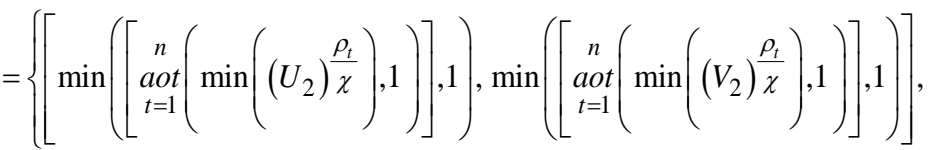

$\min \left(\left[\begin{array}{c}n \\ \operatorname{aot} \\ t=1\end{array}\left(\min \left(\left(W_{2}\right) \frac{\rho_{t}}{\chi}\right), 1\right)\right], 1\right)$

$\left[\min \left(\left[\underset{t=1}{\operatorname{aot}}\left(\min \left(\left(X_{2}\right)^{\frac{\rho_{t}}{\chi}}\right), 1\right)\right], 1\right), \min \left(\left[\underset{t=1}{\operatorname{aot}}\left(\min \left(\left(Y_{2}\right)^{\frac{\rho_{t}}{\chi}}\right), 1\right)\right], 1\right),\right]$.

$=\left\{\left[\min \left(U_{n}^{\frac{f \rho_{t}}{\chi}}, 1\right), \min \left(V_{n}^{\frac{f \rho_{t}}{\chi}}, 1\right)\right], \min \left(W_{n}^{\frac{f \rho_{t}}{\chi}}, 1\right)\right.$,

$\left.\left[\min \left(X_{n}^{\frac{f \rho_{t}}{\chi}}, 1\right), \min \left(Y_{n}^{\frac{f \rho_{t}}{\chi}}, 1\right)\right]\right\}$

Now consider, 


$$
\begin{aligned}
& f \bullet T I T 2 W A_{\rho}\left(\overline{F_{1}}, \overline{F_{2}}, \ldots, \overline{F_{n}}\right) \\
& =f \bullet\left\{\min \left(U_{n}^{\frac{\rho_{t}}{\chi}}, 1\right), \min \left(V_{n}^{\frac{\rho_{t}}{\chi}}, 1\right), \min \left(W_{n}^{\frac{\rho_{t}}{\chi}}, 1\right),\right. \\
& \left.\left[\min \left(X_{n}^{\frac{\rho_{t}}{\chi}}, 1\right), \min \left(Y_{n}^{\frac{\rho_{t}}{\chi}}, 1\right)\right]\right\} \\
& =\left\{\left[\min \left(\left(U_{n}^{\frac{f}{\chi}}\right)^{\rho_{t}}, 1\right), \min \left(\left(V_{n}^{\frac{f}{\chi}}\right)^{\rho_{t}}, 1\right)\right], \min \left(\left(W_{n}^{\frac{f}{\chi}}\right)^{\rho_{t}}, 1\right),\right. \\
& \left.\left[\min \left(\left(X_{n}^{\frac{f}{\chi}}\right)^{\rho_{t}}, 1\right), \min \left(\left(Y_{n}^{\frac{f}{\chi}}\right)^{\rho_{t}}, 1\right)\right]\right\} \\
& =\left\{\left[\min \left(\left(U_{n}^{\frac{f \rho_{t}}{\chi}}\right), 1\right), \min \left(\left(V_{n}^{\frac{f \rho_{t}}{\chi}}\right), 1\right)\right], \min \left(\left(W_{n}^{\frac{f \rho_{t}}{\chi}}\right), 1\right),\right. \\
& \left.\left[\min \left(\left(X_{n}^{\frac{f \rho_{t}}{\chi}}\right), 1\right), \min \left(\left(Y_{n}^{\frac{f \rho_{t}}{\chi}}\right), 1\right)\right]\right\}
\end{aligned}
$$

Since $(15)=(16)$, hence the result.

D. Theorem (Stability)

$$
\text { If } t>0 \quad \overline{F_{n+1}}=\left(\left[l_{\underline{F_{n+1}}}, \overline{l_{F_{n+1}}}\right], c_{F_{n+1}},\left[\underline{r_{F_{n+1}}}, \overline{r_{F_{n+1}}}\right]\right) \text { then }
$$

$\operatorname{TIT}_{2} W A_{\rho}\left(f \bullet \overline{F_{1}} \oplus \overline{F_{n+1}}, f \bullet \overline{F_{2}} \oplus \overline{F_{n+1}}, \ldots, f \bullet \overline{F_{n}} \oplus \overline{F_{n+1}}\right)$

Proof:

$$
\begin{aligned}
& \text { TIT2WA }\left(f \bullet \overline{F_{1}} \oplus \overline{F_{n+1}}, f \bullet \overline{F_{2}} \oplus \overline{F_{n+1}}, \ldots, f \bullet \overline{F_{n}} \oplus \overline{F_{n+1}}\right) \\
& =\left\{\left[\min \left(U_{n}^{\frac{f \rho_{t}}{\chi}}, 1\right) \oplus \overline{F_{n+1}}, \min \left(V_{n}^{\frac{f \rho_{t}}{\chi}}, 1\right) \oplus \overline{F_{n+1}}\right],\right. \\
& \min \left(W_{n}^{\frac{f \rho_{t}}{\chi}, 1}\right) \oplus \overline{F_{n+1}}, \\
& \left.\left[\min \left(X_{n}^{\frac{f \rho_{t}}{\chi}, 1}\right) \oplus \overline{F_{n+1}}, \min \left(Y_{n}^{\frac{f \rho_{t}}{\chi}, 1}\right) \oplus \overline{F_{n+1}}\right]\right\}
\end{aligned}
$$

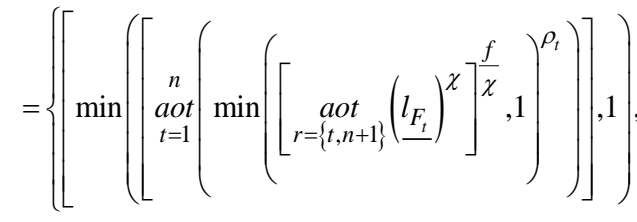

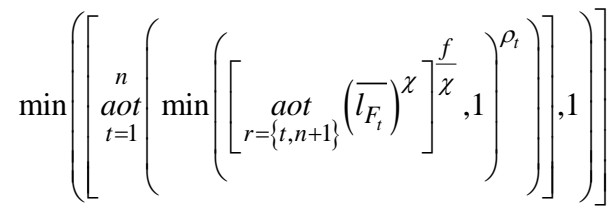$$
\min \left(\left[\underset{t=1}{\operatorname{aot}}\left(\min \left(\left[\underset{r=\{t, n+1\}}{\operatorname{aot}}\left(c_{F_{t}}\right)^{\chi}\right]^{\frac{f}{\chi}}, 1\right)^{\rho_{t}}\right)\right], 1\right)
$$

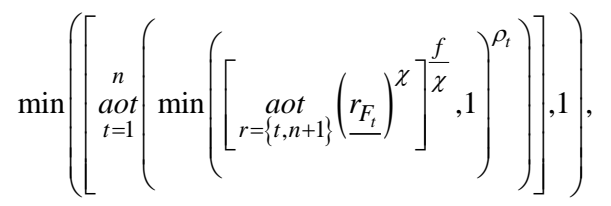

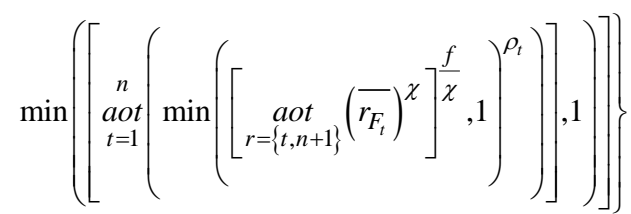$$
=\left\{\min \left(\left[U_{n}^{\frac{\rho_{t}}{\chi}}\right)+\left(\left[\left(\underline{l}_{F+1}\right)^{\chi}\right]^{\frac{1}{\chi}}\right)^{\substack{n \\ t=1}}, 1\right),\right.
$$$$
\left.\min \left(\left(V_{n} \frac{\rho_{t}}{\chi}\right)+\left(\left[\left(\overline{l_{F+1}}\right)^{\chi}\right]^{\frac{1}{\chi}}\right)^{\substack{n \\ t=1}}, 1\right)\right]
$$

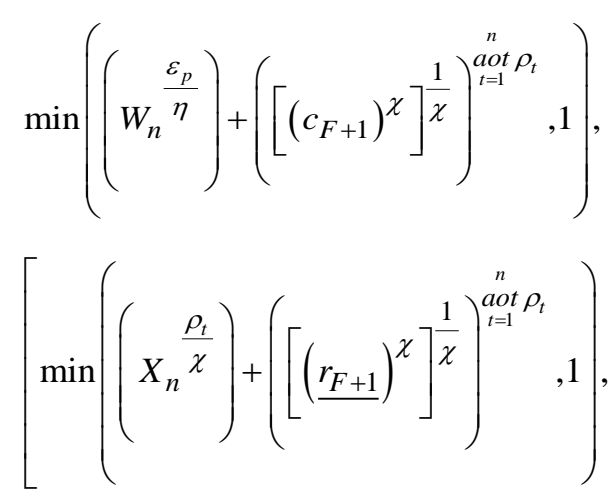




$$
\begin{aligned}
& \left.\left.\left.\min \left(\left\{Y_{n}^{\frac{\rho_{t}}{\chi}}\right)+\left(\left[\left(\overline{r_{F+1}}\right)^{\chi}\right]^{\frac{1}{\chi}}\right)^{\substack{n \\
t=t}}, 1\right]\right)\right]\right\} \\
& f \bullet T I T 2 W A_{\rho}\left(\overline{F_{1}}, \overline{F_{2}}, \ldots, \overline{F_{n}}\right) \oplus \overline{F_{n+1}} \\
& =\left\{\left[\min \left(U_{n}^{\frac{f \rho_{t}}{\chi}}, 1\right), \min \left(V_{n}^{\frac{f \rho_{t}}{\chi}}, 1\right)\right], \min \left(W_{n}^{\frac{f \rho_{t}}{\chi}}, 1\right),\right. \\
& \left.\left[\min \left(X_{n}^{\frac{f \rho_{t}}{\chi}}, 1\right), \min \left(Y_{n}^{\frac{f \rho_{t}}{\chi}}, 1\right)\right]\right\} \text {. } \\
& \oplus\left(\left[l_{F_{n+1}}, \overline{l_{F_{n+1}}}\right], c_{F_{n+1}},\left[\underline{r_{F_{n+1}}}, \overline{r_{F_{n+1}}}\right]\right) \\
& =\left\{\min \left(\left(U_{n}^{\frac{\rho_{t}}{\chi}}\right)+\left(\left[\left(\underline{l}_{F+1}\right)^{\chi}\right]^{\frac{1}{\chi}}\right), 1\right), \min \left(\left(U_{n}^{\frac{\rho_{t}}{\chi}}\right)+\left(\left[\left(\overline{l_{F+1}}\right)^{\chi}\right]^{\frac{1}{\chi}}\right), 1\right]\right. \\
& \min \left(\left(W_{n}^{\frac{\rho_{t}}{\chi}}\right)+\left(\left[\left(1-c_{F+1}\right)^{\chi}\right]^{\frac{1}{\chi}}\right), 1\right), \\
& \left.\left[\min \left(\left(X_{n}^{\frac{\rho_{t}}{\chi}}\right)+\left(\left[\left({\underline{r_{F+1}}}^{\chi}\right)^{\chi}\right]^{\frac{1}{\chi}}\right), 1\right), \min \left(\left(Y_{n}^{\frac{\rho_{t}}{\chi}}\right)+\left(\left[\left(\overline{r_{F+1}}\right)^{\chi}\right]^{\frac{1}{\chi}}\right), 1\right)\right]\right\} .
\end{aligned}
$$

Here, (18) $=(19)$ Hence the result.

\section{E. Theorem (Image Contrast)}

For given arguments $\bar{F}_{t}, t=1,2, \ldots, n$ and the parameter $\chi \in(1,+\infty)$ then TIT2WA operator is monotonically nondecreasing (MND) with respect to the parameter.

Proof:

To prove the operator is MND with respect to the parameter, we have to prove the same for every reference point function is MND w.r.t the parameter.

Since $0 \leq l_{F} \leq \bar{l}_{F} \leq c_{F} \leq \underline{r_{F}} \leq \bar{r}_{F} \leq 1 \quad \min \left(U_{5}^{\frac{k}{\chi}}, 1\right)>0$.

And it is true for all the reference points. Hence the result. $\max \left(\left(1-C_{n}^{\frac{\varepsilon_{p}}{\eta}}\right)+\left(\left[\left(1-c_{M+1}\right)^{\eta}\right]^{\frac{1}{\eta}}\right)^{\substack{n \\ \text { sum } \\ p=1}}, 0\right)$,

Based on the theorem A and the operational law,

$\operatorname{TIT}_{2 \mathrm{FYWG}_{\varepsilon}}\left\langle\overline{M_{1}}, \overline{M_{2}}, \ldots, \overline{M_{n}}\right\rangle_{\stackrel{\bullet}{\bullet} \underset{Y}{\otimes} \overline{M_{n+1}}}$

\section{APPLiCATION OF TyPe-2 FuZZy LOGIC FOR FEATURE EXTRACTION}

Fig. 2 is the proposed algorithm for Brain extraction from a DICOM image using triangular norms.

MRI of the patient DICOM image has been considered for this application, Fig. 3. Using MATLAB 2015a, brain has been extracted from MRI. The image is taken from our empirical data and its description is as follows

$\begin{array}{ll}\text { Size of the image } & : 512 \times 512 \\ \text { Mean of the image } & : 242 \times 4 \\ \text { Standard deviation } & : 50.31 \\ \text { Mean absolute deviation } & : 22.43\end{array}$

The below figures are the output of the image processing application in edge detection through triangular norms by MATLAB $2015 \mathrm{a}$.

Fig. 4 is the gradient through $x$ axis and Fig. 5 is the gradient through the y axis.

The figures reveals that the image gradient to identify the region uniformly.

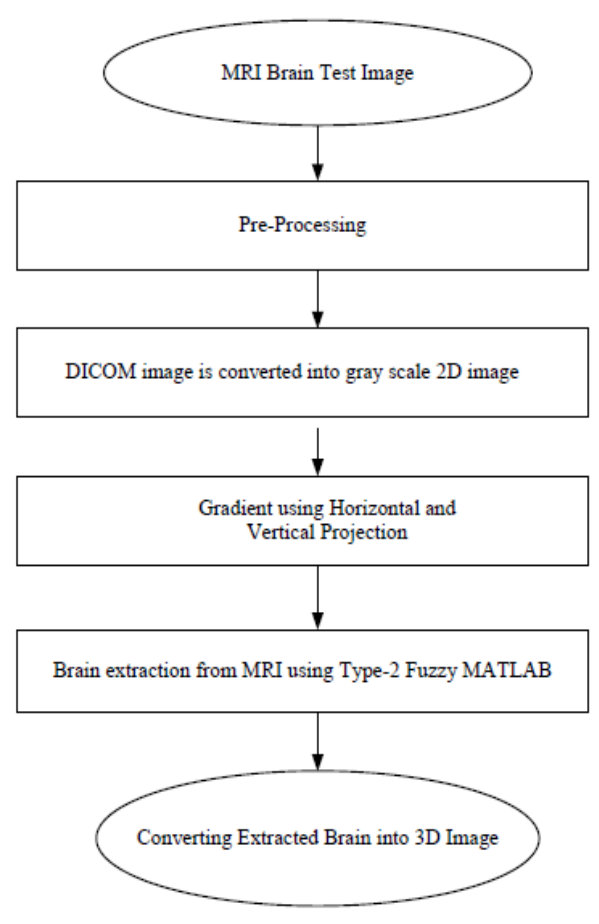

Fig. 2. Architecture of Brain Extraction. 


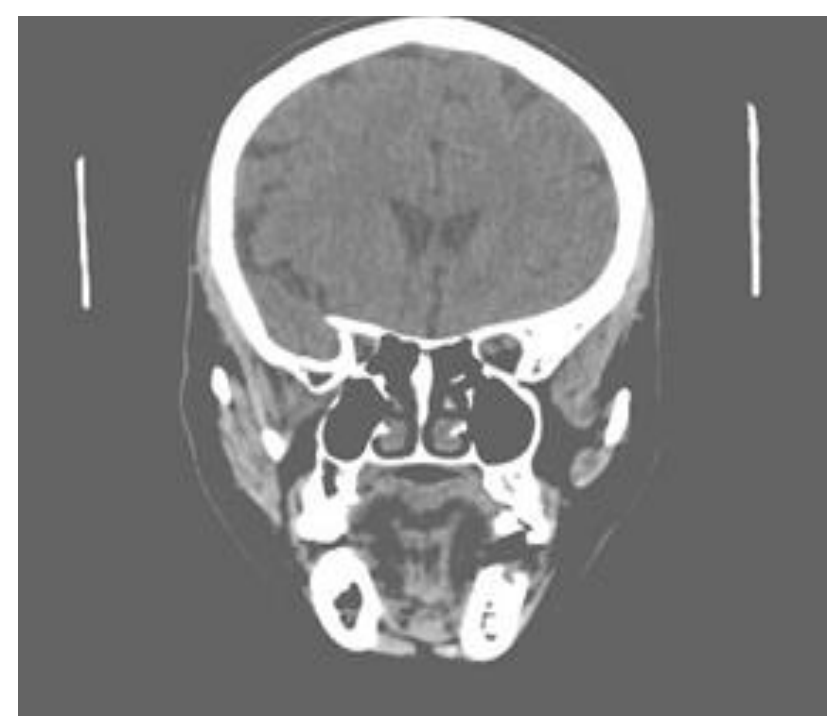

Fig. 3. Original Gray Scale DICOM Image.

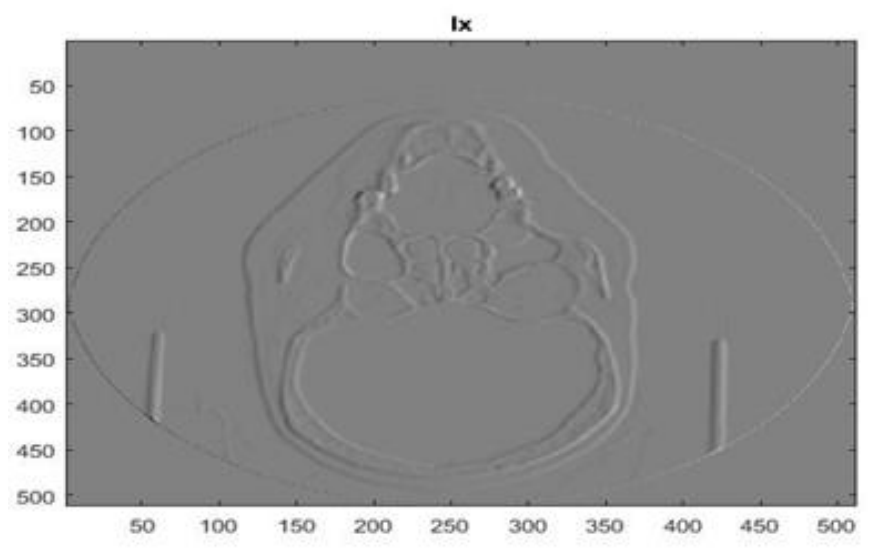

Fig. 4. Gradient through X-Axis.

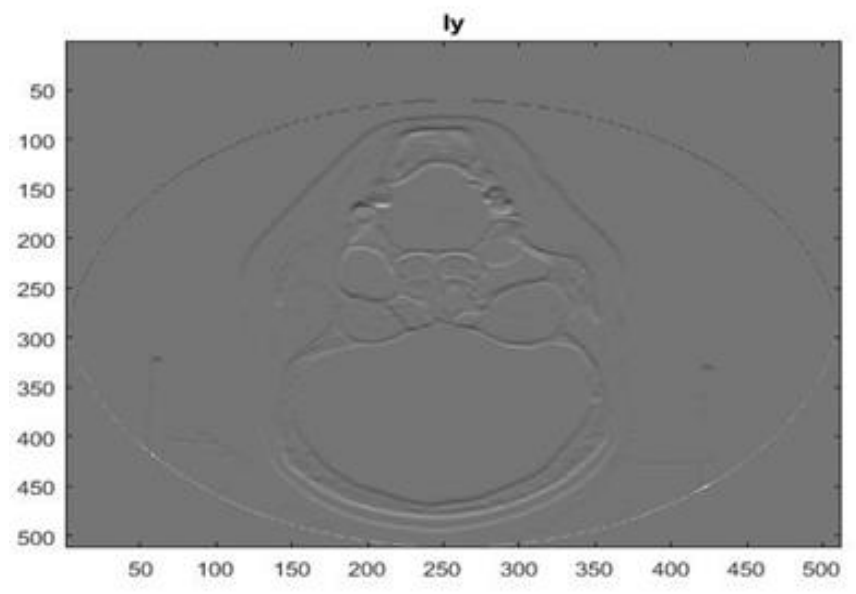

Fig. 5. Gradient through Y-axis.

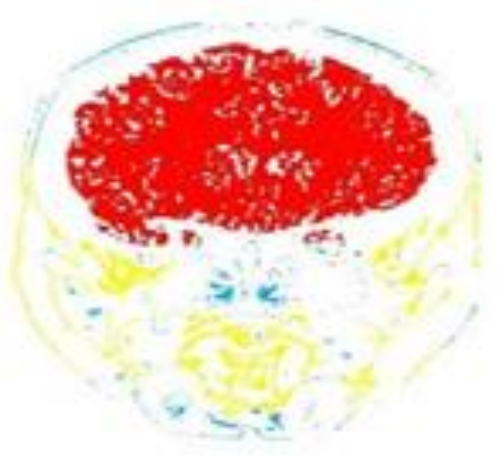

Fig. 6. Extracted Color Image.

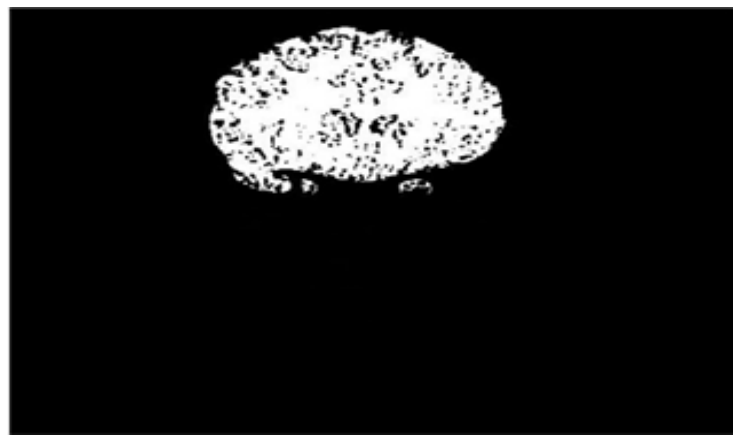

Fig. 7. Extracted Feature.

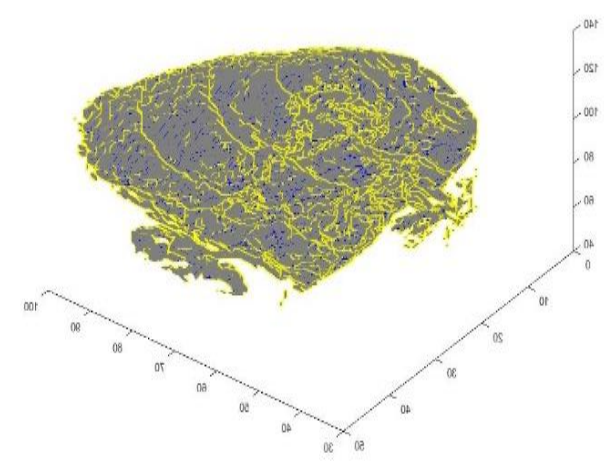

Fig. 8. 3D image of the Extracted Brain Image.

Fig. 6 is the color image output of the Brain extraction using Type-2 Fuzzy based MATLAB 2015a and Fig. 7 is the extracted brain from the DICOM image.

Fig. 8 is the 3D version of the extracted Brain image. 
Feature extraction is an essential part of the image processing. In this work, brain has been extracted from patient MRI using MATLAB 2015a. It is examined that, fuzzy logic feature extractor helps to reduce the dimensionality of the image.

Matlab coding based on Interval type-2 fuzzy logic unable to handle non-membership and indeterminacy of the feature which is to be extracted and it is the limitation of the present study.

\section{DISCUSSION}

The coding of MATLAB 2015 a under interval type-2 fuzzy has not been used to extract a feature from the image. In Literature review, previous studies have been reviewed on feature extraction and there is no contribution of work for brain extraction using the applied MATLAB coding and 3D version of the extracted color image. This shows the novelty of the proposed work.

\section{VII.CONCLUSION}

Extracting a desired feature plays a key role of image processing. This transforms the pieces from high dimensional space to low dimensional and to decrease the degree of the dimensional. Hence it helps to reduce the dimensionality of the image. In this paper, the proved mathematical properties are related to image processing especially feature extraction such as stability and image contrast and brain has been extracted from patient MRI in a better way and produced 3D image of the extracted brain using interval Type-2 fuzzy MATLAB and it is very helpful for dimensionality reduction while saving the data of the image. Using this technology, extra growth of the cells can be detected and diagnosed if any. In future this work would be extended to intuitionistic and neutrosophic environments.

Data Availability statement

The DICOM data used to support the findings of this study are available from the corresponding author upon request.

\section{Conflict of interest}

The authors declare that they have no conflict of interest.

\section{Supplementary Materials}

The data set in Fig. 9 is the montage of the images in a single file and is from a patient MRI. This MRI which is in the 3D form is converted to 2D form (DICOM) using MATLAB2015a. The 3D format consists of 25 DICOM file formats; the montage of the images is obtained as a single frame. Out of these 25 DICOM images a clear full image is chosen as in Fig. 10. Using Dilation and erosion methods, the gradient is identified. The edge detection is performed through triangular norms using MATLAB 2015a.

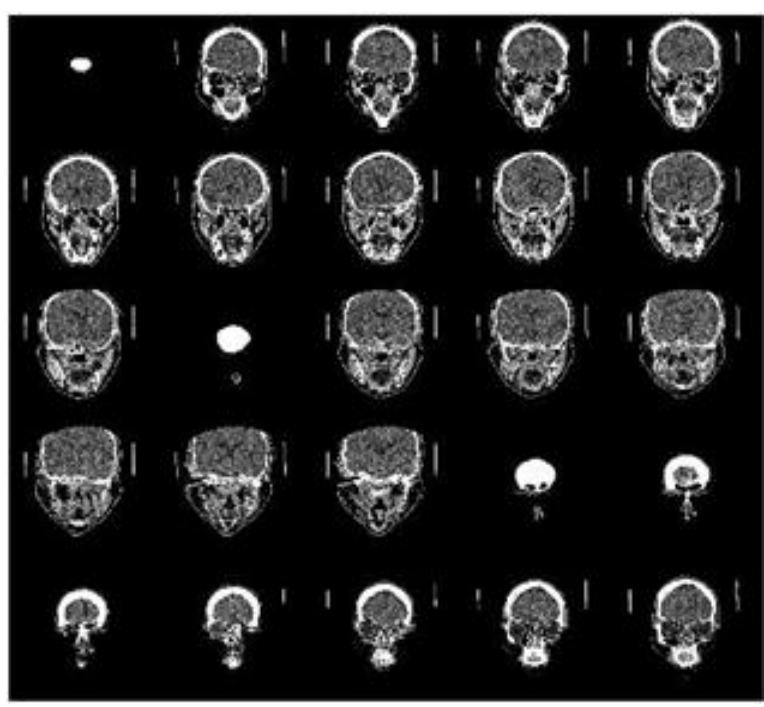

Fig. 9. Montage of the Images.

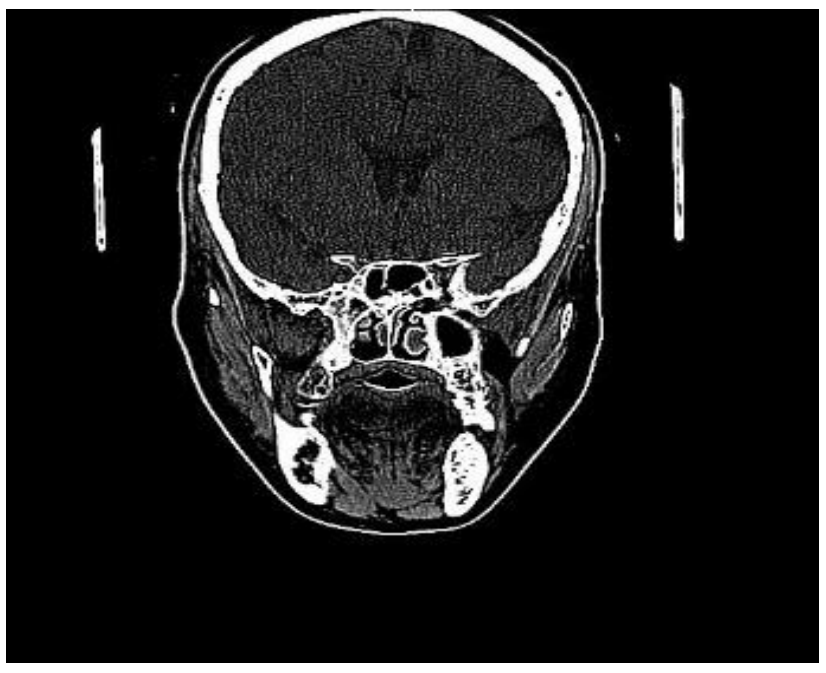

Fig. 10. Clear Image from Montage.

\section{REFERENCES}

[1] L. A. Zadeh, "The concept of a linguistic variable and its application to Approximate Reasoning-I," InformationSciences, Vol.8, No.3, 1975, pp.199-249.

[2] J. Dombi, "A general class of fuzzy operators, the DeMorgan class of fuzzy operators and fuzziness measures induced by fuzzy operators," Fuzzy Sets and Systems, Vol. 8, No.2, 1982, pp.149-163.

[3] E. Czogala and H. Zimmermann, "The Aggregation Operators for Decision Making in Probabilistic Fuzzy Environment," Fuzzy Sets and Systems, Vol.9, No.1, 1984, pp.183-196.

[4] D. Dubois and H. Prade, "A review of fuzzy set aggregation Connectives, Information Sciences,” Vol. 36, No.1-2, 1985, pp. 85-121.

[5] M. M. Gupta and J. Qi, "Theory of t-norms and Fuzzy Inference Methods," Fuzzy Sets and Systems, Vol. 40, No. 3, 1991, pp.431-450.

[6] R. R. Yager, "Aggregation operators and fuzzy systems modeling," Fuzzy Sets and Systems, Vol. 67, No.2, 1994, pp.129-145. 
[7] Y. Yao and J. Wang, "Interval based Uncertain Reasoning using Fuzzy and Rough Sets," Advances in Machine Intelligence \& Soft-Computing, 1996, pp.1-20.

[8] M. Detynieki, "Mathematical Aggregation Operators and their Application to Video Querying," Ph.D. Thesis in Artificial Intelligence Specialty of Paris, 2000, pp.1-104.

[9] K Franke, M. Koppen and B.Nickolay, "Fuzzy Image Processing by using Dubois and Prade Fuzzy Norms," Pattern Recognition, 2000, pp.14.

[10] K. Franke and M. Koppen, "A computer-based system to support forensic studies on handwritten documents," International Journal on Document Analysis and Recognition, 2000, pp.1-13.

[11] T. Calvo and R. Mesiar, "Weighted Means Based on Triangular Conorms," International Journal of Uncertain Fuzziness Knowledge Based Systems, Vol.29, No.12, 2001, pp.1173-1180.

[12] C. H. Chiu and W. J. Wang, "A simple computation of MIN and MAX operations for fuzzy numbers," Fuzzy Sets and Systems, Vol.126, No. 2, 2002, pp.273-276.

[13] D. Dubois and H. Prade, "On the Use of Aggregation operations in Information Fusion Processes," Fuzzy Sets and Systems, Vol. 142, No.3, 2004, pp.143-161.

[14] K. Hirota, H. Nobuhara, K. Kawamoto and S. I. Yoshida, "On a Lossy Image Compression/ Reconstruction Method Based on Fuzzy Relational Equations," Iranian Journal of Fuzzy Systems, Vol.1, No.1, 2004, pp.3342.

[15] S. Zhou, F. Chiclana, R. John and J. Garibaldi, "Type-1 OWA operators for aggregating uncertain information with uncertain weights induced by type-2 linguistic Quantities," Fuzzy Sets and Systems, Vol.159, No.24, 2008, pp.3281-3296.

[16] R. Mesiar, A. Kolesarova, T. Calvo and M. Komornikova, "A review of aggregation functions," In: Fuzzy Sets and Their Extensions: Representation, Aggregation and Models, H.Bustince et al. (Eds.), Springer, Berlin, 2008, pp.121-144.

[17] Z. Gera and J. Dombi, "Exact calculations of extended logical operations on fuzzy truth values," Fuzzy Sets and Systems, Vol.159, No.11, 2008, pp.1309-1326.

[18] R. Maini and H. Aggarwal, "Study and Comparison of Various Image Edge Detection Techniques," International Journal of Image Processing, Vol.3, No.1, 2009, pp.1-12.

[19] M. G. Hidalgo, A. M. Torres, D. R. Aguilera, J. T. Sastre, "Image Analysis Applications of Morphological Operators on Uninorms," Soft computing techniques for uncertainty management in image processing, 2009, pp. 630-635. ISBN: 978-989-95079-6-8.

[20] A. A. Kiaei, S. B. Shouraki, S.H. Khasteh, M. Khademi and A. R. G. Samani, "New S-norm and T-norm Operators for Active Learning Method," Fuzzy Optimization and Decision Making, 2010, pp.1-11.

[21] T. Calvo, G. Mayor and R. Messiar, "Aggregation Operators: New Trends and Application," 2012, Springer Publications.
[22] J. Qin and X. Liu, "Frank Aggregation operators for Triangular Interval Type-2 Fuzzy Set and its Application in Multiple Attribute Group Decision Making," Journal of Applied Mathematics, Vol. 1, 2014, pp.124.

[23] F. Castro-Company and P. Tirado, "On Yager and Hamacher t-norms and Fuzzy Metric spaces," International Journal of Intelligent Systems, Vol.29, No.12, 2014, pp.1173-1180.

[24] T. Chaira, "Medical Image Processing: Advanced Fuzzy Set Theoretic Techniques," CRC Publications, 2015, ISBN: 9781498700450CAT\#K24522.

[25] D. Vivona and M. Divari, "On Information Reception and Some of its Aggregation Operators," 8th International Summer School on Aggregation Operators, Kotowice, Poland, University of Silesia, 2015, pp.233-237.

[26] O. Castillo, M. A. Sanchez, C. I. Gonzalez and G. E. Martinez, "Review of Recent Type-2 Fuzzy Image Processing Applications," Information, Vol. 8, No. 97, 2017, pp. 1-18.

[27] I. Sheikh and K. A. Khan, "Simulation of Image Edge Detection using Fuzzy Logic in MATLAB," International Journal of Computer \& Mathematical Sciences, Vol. 6, No. 5, 2017, pp. 19-22.

[28] M. Lathamaheswari, D. Nagarajan, A. Udayakumar, and J. Kavikumar, "Review on Type-2 Fuzzy in Biomedicine," Indian Journal of Public Health Research \& Development.

[29] Kenjharayoobchandio and Yasarayaz, "Fuzzy Logic Based Digital Image Edge Detection," International Journal of Electrical, Electronics and Data Communication, Vol. 6, No. 2, 2018, pp.18-22.

[30] D. Nagarajan, M. Lathamaheswari, R. Sujatha and J. Kavikumar, "Edge Detection on DIOM Image using Triangular Norms in Type-2 Fuzzy," International Journal of Advanced Computer Science and Applications, Vol. 9, No. 11, pp.462-475.

[31] A. K. Dutta, "Intuitionistic Fuzzy Logic Implementation in Image Fusion Technique," Asian Journal of Research in Computer Science, Vol. 1. No. 1, 2018, pp. 1-7.

[32] M. Lathamaheswari, D. Nagarajan, J. Kavikumar and C. Phang, "A Review on Type-2 Fuzzy Controller on Control System," Journal of Advanced Research in Dynamical and Control Systems, Vol.10, No.11, 2018, pp.430-435.

[33] D. G. Privezentsev, A. L. Zhiznyakov, A. V. Astafiev and E. V. Pugin, "Using fuzzy fractal features of digital images for the material surface analysis," Journal of Physics, Vol. 944, 2018, pp. 1-5.

[34] D.Nagarajan, "Three dimensional visualization of brain using machine learning". International Journal of pure and applied mathematics, Vol:117 no.7, 2017,p.459-466.

[35] D.Nagarajan, Nagarajan.V, Abitha Gladis N.K,, "Block Processing And Edge Detection For A Dicom Image". International Journal of Pure and Applied Mathematical Sciences Vol 9,No 1, 2016,pp 9-16.

[36] D.Nagarajan, "Image denoiseing using LU decomposition and Features extraction using GLCM", International Journal of Advanced Research in Computer Science, Vol:8 no.7,2017,pp.675-677. 